\title{
Code to calculate optical properties for plasmas in a wide range of densities
}

\author{
R. Rodríguez ${ }^{1, *}$, J.M. Gil ${ }^{1}$, R. Florido ${ }^{1}$, J.G. Rubiano ${ }^{1}$, P. Martel ${ }^{1}$ \\ and $\mathrm{E}$. Mínguez ${ }^{2}$ \\ ${ }^{1}$ Departamento de Física de la Universidad de Las Palmas de Gran Canaria, \\ Campus de Tafira, 35017 Las Palmas de Gran Canaria, Spain \\ 2 Instituto de Fusión Nuclear, Universidad Politécnica de Madrid, 28006 Madrid, Spain
}

\begin{abstract}
A flexible code developed to obtain optical properties for plasmas in a wide range of densities and temperatures named ATOM3R-OP is presented. It is structured in three modules devoted to the calculation of the atomic magnitudes, the ionic abundances and the optical properties, respectively, which are briefly described. Finally, some results and remarks are shown for the source function of carbon plasmas.
\end{abstract}

\section{INTRODUCTION}

In several research fields of current interest such as astrophysics or inertial fusion confinement the knowledge and the understanding of the interactions between the photons and the plasma particles, i.e. plasma optical properties, result essential. Thus, as an example, the radiation emitted from hot plasmas or from short-life plasmas could be the most important diagnostic tool since the emitted spectrum contains information about the local instantaneous density and temperature [1]. Therefore, plasma optical properties must be determined properly which implies accurate calculations both of the atomic data and the populations of the electronic configurations involved.

In previous works [2,3] we had presented an opacity calculation code, ANALOP, which was competitive for calculating opacities for plasmas under Local Thermodynamic Equilibrium (LTE) conditions. This code generated the atomic data making use of simple atomic models based on analytical potentials into the context of the Independent Particle Model (IPM). However, it has been our aim to develop a more general and flexible code to calculate several relevant optical properties such as opacities, emissivities and source functions which is valid in a wide range of densities and temperatures covering this way both non-LTE, LTE and Corona Equilibrium (CE) situations. This new code has been named ATOM3R-OP and it is presented in this work. It is structured in three modules: the first one is devoted to the calculation of the atomic data required; in the second one the level populations are computed and in the third module the optical properties are obtained making use of the outputs of the two previous modules. In the following section the code is described and in the last section, some results and general remarks are shown.

\section{DESCRIPTION OF THE CODE}

Both the first and the second modules commented above were developed firstly in ATOM3R code [4], which provides accurate level populations for plasmas under different conditions. Therefore, these two modules will be explained more briefly than the third one.

\footnotetext{
*Address correspondences: Rafael Rodríguez, Departamento de Física, Universidad de Las Palmas de Gran Canaria, Campus de Tafira, Las Palmas de Gran Canaria 35017, Spain, e-mail: rrodriguez@dfis.ulpgc.es
} 


\subsection{Atomic data module}

Depending on the atomic number of the element under consideration, this module can work in two complementary levels. The first one is mainly used for low-Z plasmas where we use a detailed level description provided by the FAC code [5] in which are calculated the bound states of the atomic systems with convenient specification of coupling schemes and including configuration mixing. On the other hand, for intermediate and high-Z plasmas this detailed calculation becomes impracticable and the use of analytical potentials, into the IPM context, becomes an interesting and useful method to work out accurate ionic populations and average ionizations in a wide range of densities and temperatures. In particular, ATOM3R-OP operates using a set of analytical potentials developed by us which include plasma effects [6] and single and core excited configurations [7]. For situations wherein more celerity is desired, the code can perform the atomic calculations using a relativistic screened hydrogenic model [8].

\subsection{Level populations module}

Using as an input the atomic data provided by the later module, in this one fast calculation of ionic charge state distributions and level populations are performed by means of solving a collisional-radiative steady-state model (CRSS) including the continuum lowering through the Stewart and Pyatt expression. The populations are computed with a reasonable accuracy for plasmas of any element in a wide range of conditions embracing non-LTE, LTE and CE situations and it is valid both for optically thin and thick plasmas [9]. In the last case, the reabsorption of the radiation is modeled through the escape factor formalism [10]. The processes included in the CRSS are the following: collisional excitation and desexcitation; spontaneous decay; collisional ionization and three body recombination; radiative recombination and dielectronic recombination, being most of the rate coefficients evaluated by analytical formulas that can be found in the literature. In order to solve the set of rate equations, we employee the technique of sparse matrix to storage the non-zero elements of the equations system. This implies substantial savings in computing time and memory requirements and it also allows us to include a large amount of ionic configurations in our calculations. On the other hand, this fact increases the system dimension, which can easily reach the order of $10^{5}$ for low-Z plasmas, and the most suitable choice to carry out the matrix inversion are the iterative procedures [5] because they require much less memory than direct methods and also they are faster. It is worth standing out this last fact because when we include plasma effects in the plasma model this module and the atomic one have to be solved iteratively until the convergence is achieved.

\subsection{Optical properties module}

This module is devoted to determine the emissivity, opacity and source function making use of the populations and the atomic data given in the previous modules. The bound-bound opacity and emissivity are calculated by

$$
\begin{gathered}
\kappa_{v}^{b b}=\frac{1}{\rho} \frac{\pi}{m c} \frac{e^{2}}{4 \pi \varepsilon_{0}} \sum_{\zeta} \sum_{i j} n_{i}^{\zeta} f_{i j}^{\zeta} \varphi^{\zeta}\left(v-v_{i j}\right)\left[1-\frac{n_{j}^{\zeta} g_{i}^{\zeta}}{n_{i}^{\zeta} g_{j}^{\zeta}}\right] \\
j_{v}^{b b}=\frac{e^{2} h v^{3}}{2 \varepsilon_{0} m c^{3}} \sum_{\zeta} \sum_{i j} n_{j}^{\zeta} \frac{g_{i}^{\zeta}}{g_{j}^{\zeta}} f_{i j}^{\zeta} \varphi^{\zeta}\left(\nu-v_{i j}\right)
\end{gathered}
$$

where $\zeta$ denotes the ionic state and $i j$ and $f_{i j}^{\zeta}$ the line transition between the configurations $i$ and $j$ and its oscillator strength, respectively. $n_{i}^{\zeta}$ and $g_{i}^{\zeta}$ are the population and the statistical weight of the electron 
configuration $i$, respectively. $\varphi$ is a voigt profile. It has been assumed that is the same both for emission and absorption. Finally, $\rho$ is the density of matter, $c$ the speed of the light and $m$ the mass of the electron. The bound-free spectrum is calculated making use of the cross sections calculated by using FAC code [5] which are interpolated subsequently to our photon energy mesh. They are calculated through accurate methods avoiding the semi-classical Kramer's formula which introduce high errors when evaluating non-hydrogenic states [11]. In terms of the cross sections the bound-free opacity and emissivity are evaluated as

$$
\begin{gathered}
\kappa_{v}^{b f}=\frac{1}{2 \rho}\left(\frac{h^{2}}{2 m \pi k T}\right)^{3 / 2} \sum_{\zeta} \sum_{i j} n_{i}^{\zeta}\left[1-\frac{n_{j}^{\zeta+1} n_{e} e^{-\left(h v-E_{T}^{\zeta, i}\right) / k T}}{n_{i}^{\zeta}} \frac{g_{i}^{\zeta}}{g_{j}^{\zeta+1}}\right] \sigma_{\zeta+1, j}^{\zeta, i_{i}}(v) \\
j_{v}^{f b}=\frac{h v^{3}}{c^{2}}\left(\frac{h^{2}}{2 m \pi k T}\right)^{3 / 2} n_{e} \sum_{\zeta} \sum_{i j} n_{j}^{\zeta+1} \frac{g_{i}^{\zeta}}{g_{j}^{\zeta+1}} e^{-\left(h v-E_{T}^{\zeta, i}\right) / k T} \sigma_{\zeta+1, j}^{\zeta, i_{i}}(v)
\end{gathered}
$$

where $T$ is the plasma temperature, $k$ is the Boltzmann's constant, $n_{e}$ is the free electron density, $E_{T}^{\zeta, i}$ is the threshold energy of configurations $i$ and $\sigma(v)$ is the bound-free cross section. It has been assumed a Mawell-Boltzmann distribution for the free electrons. Finally, the free-free spectrum has been obtained employing the Kramer's approximation for the cross section (corrected by the gaunt factor) assuming that the velocity distribution is Maxwellian. Once both the emissivity and the opacity have been calculated the source function is estimated as the ratio between the total emissivity and the linear extinction coefficient which is obtained from the total opacity multiplies by the density of matter.

\section{RESULTS AND GENERAL REMARKS}

As it is known, the source function for plasmas under LTE conditions behaves like the Plank distribution. This fact allows us to check our model since our source function should tend to the Planck function as the plasma conditions are closer to the LTE situations. In figures 1 and 2 are shown the source functions calculated for optically thin carbon plasmas at the same density $10^{20} \mathrm{~cm}^{-3}$ but at two temperatures, 25 and

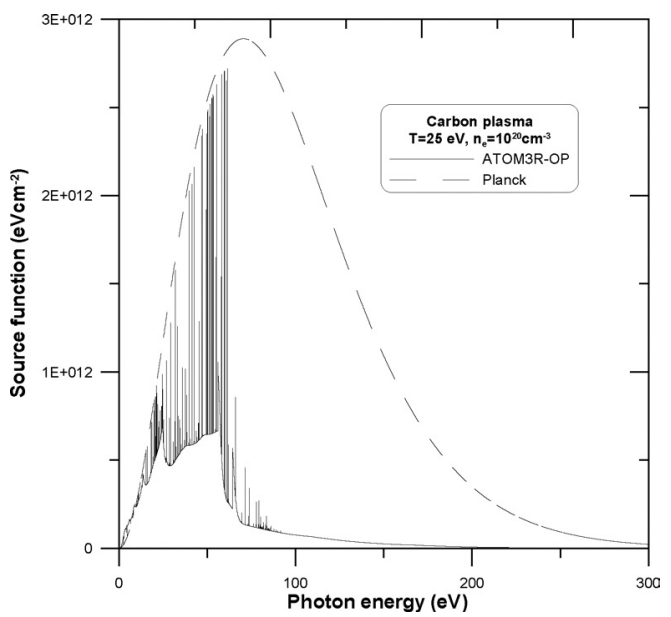

Figure 1. Source function for carbon plasma under NLTE conditions.

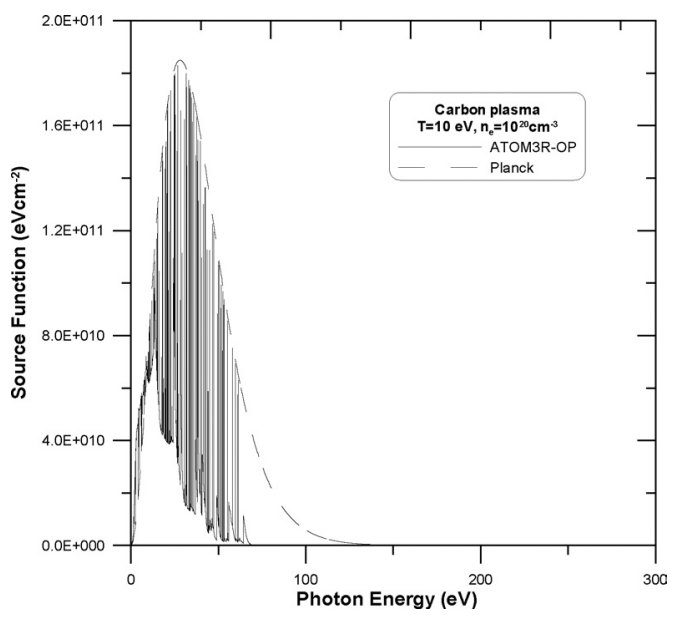

Figure 2. Source function for carbon plasma under LTE conditions. 
$10 \mathrm{eV}$, being their average ionization 3.91217 and 3.26578, respectively. In both situations the number of bound configurations involved is higher than 850 giving as result more than 10000 bound-bound transitions and 21000 bound-free transitions. The results are compared with the Planckian distribution. The situation of lower temperature (figure 2) is nearer to LTE conditions than the other one (figure 1) and a good agreement is found with respect to the Planck distribution. However, it can be also observed from figure 2 that there are some discrepancies between our results and the Planck distribution for photons with energy higher than $60 \mathrm{eV}$, being our source function smaller than the Planckian one in this range of energies. These differences are probably due to in our atomic model we have not included the doubly excited states of the carbon ions and the transitions associated to them, which lies on this range of photon energies, are being neglected.

\section{Acknowledgments}

This work has been supported by the Project of the Spanish "Ministerio de Educación y Ciencia" with reference ENE2004-08184-C03-01/FTN, by the Project UNI22/2003 of "Las Palmas de Gran Canaria University" and by the program "Keep in Touch" of the "European Union".

\section{References}

[1] Salzmann D., Atomic Physics in Hot Plasmas (Oxford University Press, New York, 1998).

[2] Mínguez E., Gil J.M., Martel P., Rubiano J.G., Rodríguez R., Doreste L., Nucl. Instr. and Meth. in Phys. Res. A 415 (1998), pp. 539-542.

[3] Rubiano J.G., Florido R., Rodríguez R., Gil J.M., Martel P., Mínguez E., J. Quant. Spectrosc. Radiat. Transfer 83 (2004) 159-182.

[4] Florido R., Gil J.M., Rodríguez R., Rubiano J.G., Martel P., Mínguez E., "ATOM3R, a code for calculation of NLTE plasma populations. Low and high density behavior." Proceedings of the 28th Internacional Conference on Laser Interaction with Matter, Rome, 2004 (XXVIII ECLIM Conference, Rome, 2005).

[5] Gu M.F., Astrophys. J. 582 (2003) 1241-1250.

[6] Gil J.M., Martel P., Mínguez E., Rubiano J.G., Rodríguez R., Ruano F.H., J. Quant. Spectrosc. Radiat. Transfer 75 (2002) 539-557.

[7] Rodríguez R., Rubiano J.G., Gil J.M., Martel P., Mínguez E., Florido R., J. Quant. Spectrosc. Radiat. Transfer 75 (2002) 723-739.

[8] Rubiano J.G., Rodríguez R., Gil J.M., Ruano F.H., Martel P., Mínguez E., J. Quant. Spectrosc. Radiat. Transfer 72 (2002) 575-588.

[9] Mínguez E., Rodríguez R., Gil J.M., Sauvan P., Florido R., Rubiano J.G., Martel P., Mancini R., Laser Part. Beams 23 (2005) 199-203.

[10] Mancini R., Joyce RF., Hooper Jr C.F., Phys. B.: At. Mol. Phys. 20 (1987) 2975-2987.

[11] Rodríguez R., Gil J.M., Florido R., Rubiano J.G., Martel P., Mínguez E., J. Quant. Spectrosc. Radiat. Transfer 91 (2005) 393-413. 